\title{
A transcranial magnetic stimulation study on response activation and selection in spatial conflict
}

ARTICLE in EUROPEAN JOURNAL OF NEUROSCIENCE - DECEMBER 2014

Impact Factor: $3.18 \cdot$ DOI: $10.1111 /$ ejn.12803

READS

71

4 AUTHORS:

Lara Bardi

Ghent University

17 PUBLICATIONS 138 CITATIONS

SEE PROFILE

\section{Demis Basso}

Libera Università di Bozen-Bolzano

38 PUBLICATIONS 327 CITATIONS

SEE PROFILE

\section{Sami Schiff}

University-Hospital of Padova

96 PUBLICATIONS 742 CITATIONS

SEE PROFILE

Daniela Mapelli

University of Padova

64 PUBLICATIONS $\mathbf{1 , 1 5 4}$ CITATIONS

SEE PROFILE 


\title{
A transcranial magnetic stimulation study on response activation and selection in spatial conflict
}

\author{
Lara Bardi, ${ }^{1,2}$ Sami Schiff, ${ }^{3}$ Demis Basso ${ }^{4}$ and Daniela Mapelli ${ }^{2}$ \\ ${ }^{1}$ Department of Experimental Psychology, Ghent University, Henri Dunantlaan 2, B-9000 Gent, Ghent, Belgium \\ ${ }^{2}$ Department of General Psychology, University of Padua, Padua, Italy \\ ${ }^{3}$ Department of Medicine, University of Padua, Padua, Italy \\ ${ }^{4}$ Faculty of Education, Free University of Bolzen-Bolzano, Bolzen-Bolzano, Italy
}

Keywords: premotor cortex, simon task, timing, transcranial magnetic stimulation

\begin{abstract}
In choice reaction tasks, subjects typically respond faster when the relative spatial positions of stimulus and response correspond than when they do not, even when spatial information is irrelevant to the task (e.g. in the Simon task). Cognitive models attribute the Simon effect to automatic response activation elicited by spatial information, which facilitates or competes with the controlled selection of the correct response as required by task demands. In the present study, we investigated the role of the dorsal premotor cortex (PMd) in response activation and selection during spatial conflict. We applied single-pulse transcranial magnetic stimulation (TMS) to the PMd of the right and left hemispheres during the execution of a Simon task, at different times after the onset of the visual stimulus. The results showed that TMS produced a different effect on subjects' performance in two separate time windows. When TMS was applied at an early time [160-ms stimulus onset asynchrony (SOA)], we observed suppression of the Simon effect, resulting from a delay of corresponding trials. When TMS was applied at a late time (220 and 250-ms SOA), we observed an increase in the Simon effect, resulting from a delay of non-corresponding trials. These outcomes revealed that the PMd is involved both in the activation of the spatially triggered response and in response selection during spatial conflict.
\end{abstract}

\section{Introduction}

Upon encountering stimuli that present action affordances, humans are faced with an action selection problem. In some situations, irrelevant visual information may activate incorrect motor tendencies with respect to the current goals, and control mechanisms are necessary to inhibit stimulus-driven responses. Therefore, response selection involves the interplay between facilitatory and inhibitory processes.

In the Simon task, a conflict arises because irrelevant spatial information competes for response selection with task-relevant information. As a result, responses are usually faster and more accurate when stimulus and response positions correspond than when they do not (Simon \& Rudell, 1967; Umiltà \& Nicoletti, 1990; Lu \& Proctor, 1995; Hommel, 2011).

Dual-route models (Kornblum et al., 1990; De Jong et al., 1994) explain conflict by postulating two parallel routes of information processing: irrelevant stimulus position primes the spatially corresponding response through a fast, direct route, while the task-relevant stimulus code activates the intention-guided response via a slow, controlled, indirect route (Kornblum et al., 1990; Wascher et al., 2001). The activation-suppression model (Ridderinkhof, 2002) refined dual-route models of interference by incorporating

Correspondence: Lara Bardi, ${ }^{1}$ Department of Experimental Psychology, as above. E-mail: lara.bardi@ugent.be

Received 27 March 2014, revised 9 November 2014, accepted 12 November 2014 specific hypotheses about the temporal dynamics of incorrect response activation and its correction: active suppression of conflicting responses needs time to become effective. Therefore, slower reaction times (RTs) are less vulnerable to impulsive actions that are captured by irrelevant stimulus dimension than faster RTs (e.g. De Jong et al., 1994; Hommel, 1994; Mapelli et al., 2003; Vallesi et al., 2005).

Neuroimaging studies have shown that the activation and selection of potential response programs involve a large fronto-parietal network. The premotor cortex (PMC) receives projections mostly from the posterior parietal cortex, and this posterior parietal cortexPMC circuit in the dorsal stream of the visual processing system has been suggested to be essential for spatial visuo-motor transformation (Stürmer et al., 2002; Iacoboni \& Zaidel, 2004). On the other hand, the PMC is recruited in action selection, in response preparation of non-standard stimulus-response mappings, or when an already planned movement has to be overcome (Wise et al., 1996; Schluter et al., 1998; Praamstra, 1999; Ridderinkhof, 2002; Egner \& Hirsch, 2005; Kerns, 2006; Koch et al., 2006; Egner et al., 2007).

In summary, previous studies have suggested that the PMC might play a critical role both in response activation and response selection during response conflict. However, this hypothesis has never been tested directly. Moreover, the dynamics of these two processes have never been explored, and have only been inferred from analysis of the lateralized electroencephalographic movement-related potentials 
(Valle-Inclán, 1996; Stürmer et al., 2002; Stürmer \& Leuthold, 2003; Vallesi et al., 2005).

In the present study, we applied single-pulse transcranial magnetic stimulation (TMS) to the PMC while participants were engaged in a Simon task. When applied at an early time after stimulus presentation, TMS was expected to induce suppression of the Simon effect, owing to interference with the planning of the spatially corresponding response. Conversely, according to the activation-suppression model, when stimulation was applied at a later time, we expected an increase in the Simon effect, owing to interference with the selection of the appropriate response in non-corresponding trials.

\section{Materials and methods}

\section{Participants}

Eighteen healthy undergraduate students of the University of Padua (all right-handed; mean age, of $23 \pm 2$ years) with normal or corrected-to-normal visual acuity took part in this experiment. The study conformed with the 2013 World Medical Association Declaration of Helsinki. All subjects were checked for TMS exclusion criteria and gave their written informed consent before participation. The intensity of stimulation and the duration of the TMS sessions were in accordance with the safety guidelines established by Wassermann (1998), and the Ethics Committee of the Department of General Psychology, University of Padua, approved the procedure. The subjects were reimbursed with cash for participating in the experiment. All participants were naive as to the purpose of the study.

\section{Apparatus and stimuli}

The participants were seated in a dimly lit and soundproof room with the head held by a fixed head-and-chin rest at a distance of $50 \mathrm{~cm}$ from a 17-inch monitor controlled by a Pentium 4 PC programmed with E-PRIME (Psychological Software Tools, Pittsburgh, PA, USA). The targets were the letters ' $\mathrm{N}$ ' and ' $\mathrm{H}$ ' subtending $1.4 \times 1.8^{\circ}$ (width $\times$ height) of a visual angle presented for $200 \mathrm{~ms}$ in white on a black background, $3.5^{\circ}$ laterally with respect to a fixation cross.

\section{Procedure and experimental design}

Each participant was tested in two experimental sessions lasting for $\sim 1.5 \mathrm{~h}$ each. The participants completed a block of 40 practice trials, followed by the experimental blocks. A trial started with the presentation of a central fixation cross (subtending $0.5 \times 0.5^{\circ}$ of visual angle) for $400 \mathrm{~ms}$. Then, a stimulus was presented for $200 \mathrm{~ms}$ at a visual angle of $4.6^{\circ}$, either to the left or to the right of the central fixation point. A contralateral filler '\#' was also presented. The maximum response time was $1200 \mathrm{~ms}$. The inter-trial interval varied randomly between 1500 and $2500 \mathrm{~ms}$. After an incorrect response, a tone $(600 \mathrm{~Hz})$ was delivered to provide feedback on the wrong response. At the end of each block, feedback concerning mean RT and accuracy was displayed on the screen. A schematic representation of the trial sequence is shown in Fig. 1B. The participants were engaged in a two-alternative choice reaction task, and were instructed to maintain their gaze on the fixation point during each block. Half of the participants were asked to respond, as quickly as possible, by pressing the leftmost key $(\mathrm{Z})$ on the keyboard with the index finger of the left hand when the target letter was an ' $\mathrm{N}$ ', and the rightmost key ' $M$ ' with the right hand when it was an ' $H$ '. Letters on the keyboard were covered with white stickers. The other half of the participants received the opposite hand-target assignment.

Each experimental session was divided into six experimental blocks, two for each stimulation site. Each block was composed of 320 trials in which stimulus position (left or right), response position (left or right) and TMS stimulus onset asynchrony (SOA) (delay of the TMS pulse from stimulus onset: 100, 130, 160, 190, 220, 250, 280 and $310 \mathrm{~ms}$ ) were fully crossed to produce the same number of trials for each possible combination, presented in a random order. A pause was given to the participants every 160 trials. Three blocks [one for each stimulation site: left dorsal PMC (PMd), right PMd, and the control site] were administered on the first day, and the remaining three blocks on the second day. The order of blocks (i.e. site of stimulation) was counterbalanced across participants.

\section{Localization of brain targets for TMS}

The location of each subject's PMd was first determined by referencing it to the individual motor hand area. The motor cortex 'hot spot' was determined as the optimal scalp position at which the lowest TMS intensity evoked a visually detectable twitch in the thumb muscle of the contralateral hand. This site was then marked on the scalp. The PMd site was marked $2 \mathrm{~cm}$ anterior and $1 \mathrm{~cm}$ medial to the motor 'hot spot'. This procedure for targeting the PMd has been used in a number of previous studies, which have shown that single-pulse TMS at these coordinates slows RTs on response choice tasks (Schluter et al., 1998; Johansen-Berg et al., 2002; O'Shea et al., 2007). The cortical location of this site was anatomically verified on T1-weighted magnetic resonance imaging scans of 10 participants, with Brainsight frameless stereotaxy (Rogue Research, Montreal, QC, Canada). Magnetic resonance images were then registered to the Montreal Neurological Institute template (Evans et al., 1996). This confirmed that TMS was applied just anterior to the dorsal branch of the precentral sulcus, with the following mean Talairach coordinates for the stimulated positions: $x= \pm 30 y=-5$, and $z=67$ (standard errors were 1.23, 2.07, and 2.05, respectively). This set of coordinates is compatible with published probabilistic coordinates for the PMd (e.g. Schluter et al., 2001; O'Shea et al., 2007). In order to control for non-specific effects of TMS, the point on the scalp corresponding to the EEG electrode $\mathrm{CPz}$ (according to the 10-20 international system for EEG electrodes placement) was stimulated as a control site.

\section{TMS parameters}

A 70-mm figure-of-eight stimulation coil was placed over the stimulation sites tangentially to the skull, with the handle pointing backwards parallel with the midline. TMS was applied with a Magstim Super Rapid2 system (Magstim Company, Whitland, UK). The intensity of the magnetic stimulation was set separately for each participant $10 \%$ above the individual motor threshold. The mean stimulating intensity was approximately $65 \pm 1.6 \%$ of the maximum stimulator output.

\section{Results}

The mean accuracy rate was 0.94 . The accuracy rate was 0.95 in the corresponding condition and 0.93 in non-corresponding trials. A repeated-measures ANOVA was performed on mean RTs, with stimulation site (left PMd, right PMd, and control), TMS SOA (100, 130, $160,190,220,250,280$ and $310 \mathrm{~ms}$ ), response side (right and left hand) and correspondence (corresponding and non-corresponding 

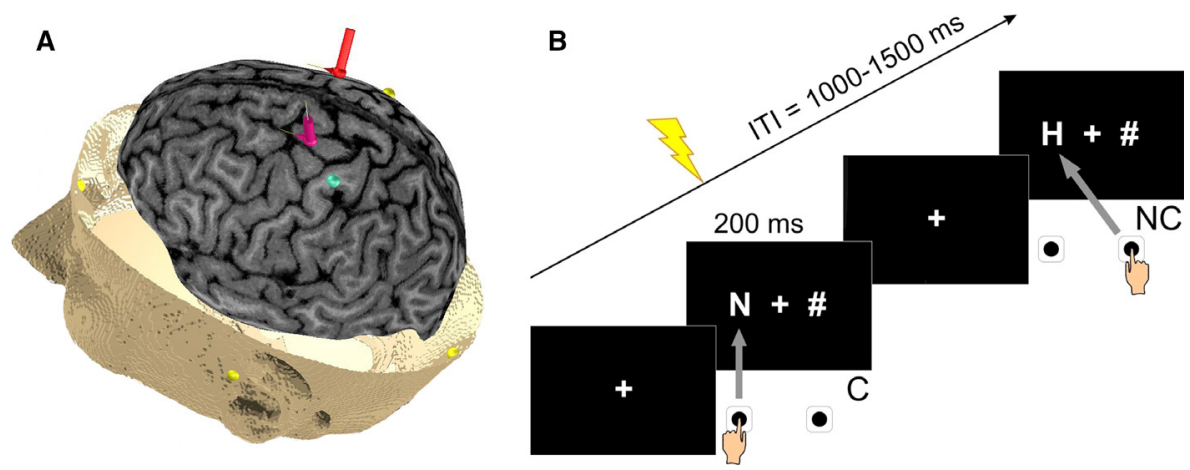

FIG. 1. (A) The 3D magnetic resonance imaging (MRI) brain reconstruction of a representative subject. The motor hot spot revealed by functional localization (posterior marker) and the left and right PMd sites (anterior trajectories) are marked on the brain image. (B) A schematic representation of the trial sequence of the Simon task. The single-pulse TMS was randomly delivered in each trial at a different SOA $(100,130,160,190,220,250,280$ and $310 \mathrm{~ms})$ with respect to stimulus presentation, whereas the inter-trial interval (ITI) randomly varied between 1000 and $1500 \mathrm{~ms}$. C, corresponding; NC, non-corresponding.

trials) as within-subject factors. Duncan's post hoc tests were performed in order to explore the significant interaction. A main effect of correspondence was found $\left(F_{1,17}=44.77, P<0.05, \eta_{\mathrm{p}}^{2}=0.72\right)$, corresponding trials (mean $\pm \mathrm{SE}$ of the mean, $469 \pm 7 \mathrm{~ms}$ ) being faster than non-corresponding trials (mean $\pm \mathrm{SE}$ of the mean, $486 \pm 7 \mathrm{~ms}$ ). Moreover, as commonly reported, the Simon effect (corresponding minus non-corresponding trials) was larger for the right hand than for the left hand, as attested by the correspondence $\times$ response side interaction $\left(F_{1,17}=19.16, \quad P<0.05\right.$, $\left.\eta_{\mathrm{p}}^{2}=0.53\right)$. More interestingly for the present study, a three-way interaction, i.e. site $\times$ TMS SOA $\times$ correspondence $\left(F_{1,238}=2.23\right.$, $\left.P<0.05, \eta_{\mathrm{p}}^{2}=0.12\right)$ was found. Corrected post hoc tests were performed on RTs for the left PMd and right PMd as compared with the control site, separately on corresponding and non-corresponding trials. The analysis revealed that TMS over the left PMd affected mean RTs when applied at three specific time points. At an early time $(160 \mathrm{~ms})$, TMS induced a delay of RTs in the corresponding condition as compared with the control $(P<0.05)$, leading to suppression of the Simon effect. When applied at later times (220 and $250 \mathrm{~ms})$, TMS affected conflict trials (namely, the non-corresponding condition). RTs in the non-corresponding condition were significantly slower than those at the control site $(P<0.05)$, resulting in an increase in the Simon effect (Fig. 2). No interaction between site and response side was found.

\section{Discussion}

The aim of the present study was to investigate the role of the PMd in response activation and selection during spatial conflict.

The main result obtained in this study showed that single-pulse TMS applied to the left PMd $160 \mathrm{~ms}$ after stimulus presentation induced suppression of the Simon effect. At later times (220 and $250 \mathrm{~ms}$ ), TMS induced an increase in the Simon effect. This effects have been also qualified in terms of correspondence conditions for both timings: the former effect was attributable to an increase in RTs in corresponding trials, whereas the latter effect was attributable to an increase in RTs in non-corresponding trials.

The specific effect obtained in corresponding trials at the early time suggests that left PMd TMS interfered with response priming driven by stimulus spatial information during the Simon task. Interestingly, the timing of this TMS effect is compatible with that obtained in a previous study on the PPC (Schiff et al., 2011). In this study, singlepulse TMS was applied to the angular gyri (AGs) and supramarginal gyri (SMGs) of the PPCs of both hemispheres while participants were engaged in a Simon task. The Simon effect was suppressed when TMS was applied to the right and left AGs, respectively, 130 and
$160 \mathrm{~ms}$ after the onset of the visual stimulus. Furthermore, suppression of the Simon effect was produced when single-pulse TMS was delivered over the left SMG, $160 \mathrm{~ms}$ after stimulus onset, but not when it was applied over the right SMG. In accordance with previous studies, the effect on bilateral AGs indicated that these regions are essential for processing the spatial code of the stimulus (e.g. Ashbridge et al., 1997), whereas the effect on the left SMG suggested that this area is involved in transforming the spatial code into action code (motor attention) (e.g. Rushworth et al., 2001, 2003). Taken together, these results are in line with the idea that the PPC-PMC circuit is a neural correlate of visuo-motor transformation through the direct pathway of information processing (De Jong et al., 1994; Stürmer et al., 2007).

The second finding is that TMS caused an increase in the Simon effect, owing to a delay in non-corresponding trials, when applied at $220 \mathrm{~ms}$. This result supports the hypothesis that the PMd is recruited during response selection when a conflict between competing responses occurs. In non-corresponding trials, the response primed by the irrelevant stimulus code does not match that activated on the basis of task instruction. Thus, the corresponding response has to be inhibited for the execution of the correct response. The present finding that temporary interference with the PMd induced an increase in the conflict supports the hypothesis that the left PMd mediates this process in a late time window. Our findings are consistent with models of conflict that postulate two parallel routes of information processing: a fast, direct route, through which the location of the stimulus primes the corresponding response; and a slow, indirect route, through which the response is activated on the basis of task instructions (Kornblum et al., 1990; De Jong et al., 1994).

Future experiments should determine whether the PMd is the source of this executive selection or, more plausibly, receives topdown control from other frontal areas related to conflict resolution, such as the inferior frontal gyrus and the dorsolateral prefrontal cortex (Ridderinkhof et al., 2004; Forstmann et al., 2008; van den Wildenberg et al., 2010; Duqué et al., 2012). In effect, it is also conceivable that the specific role of the PMd is better defined as an interface between separate systems, namely the parietal cortex, which codes for spatial information, and the frontal network, which guides correct response execution and the motor system. Importantly, the role of the PMd should not be reduced to action programming or execution (Schluter et al., 1998; Johansen-Berg et al., 2002; Mochizuchi et al., 2005). For example, in the study of Schluter et al. (1998), single-pulse TMS was applied to the PMC (with the same localization procedure as used in the present study) while participants were tested in both a choice RT task and a simple RT 

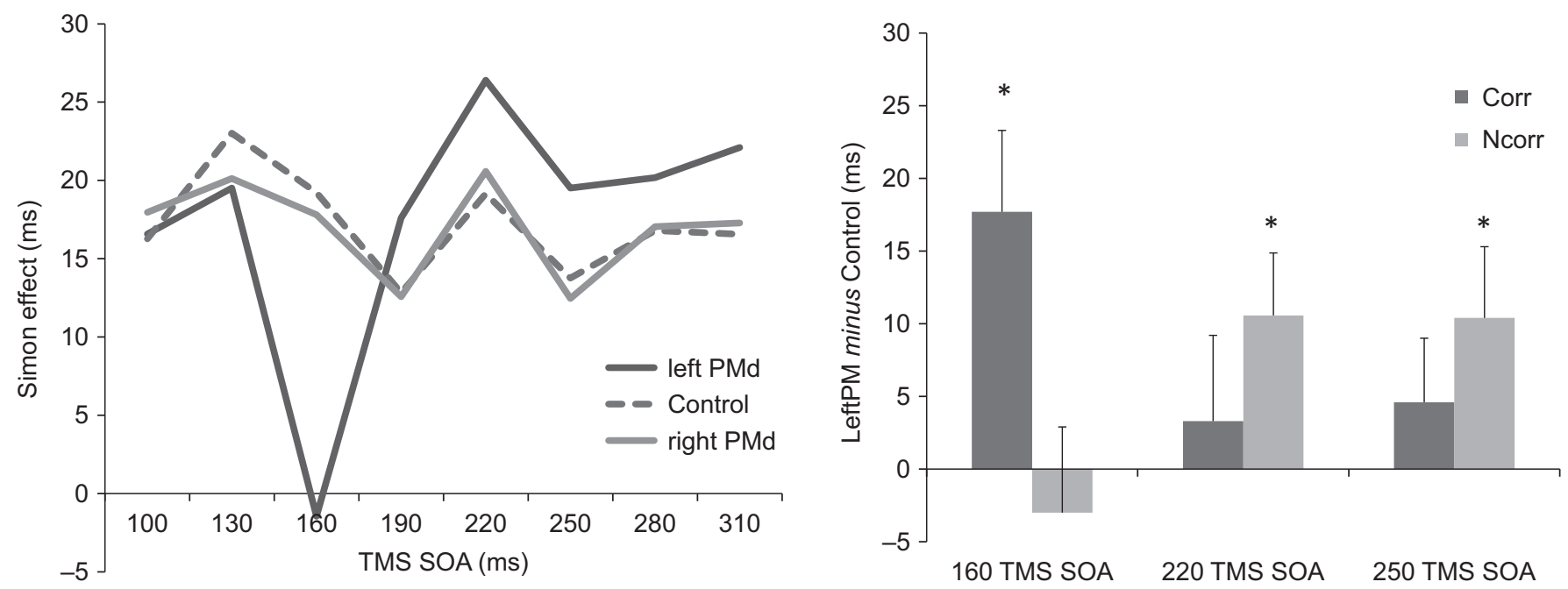

FIG. 2. Left panel: the size of the Simon effect (non-corresponding minus corresponding trials) for the left PMd, control site and right PMd is represented as a function of the TMS SOAs. Right panel: differences in mean RTs between the left PMd and the control site for corresponding and non-corresponding trials (light gray and dark gray, respectively). Left: 160-ms TMS SOA. Middle: 220-ms TMS SOA. Right: 250-ms TMS SOA. C, corresponding; N, non-corresponding. ${ }^{*} P<0.05$.

task. TMS induced a delay of RTs in the choice RT task but not in the simple RT task, suggesting that this area is involved in response selection but not in response programming when movement selection demands of the task are kept to a minimum.

The timing of our TMS effects is also compatible with previous electroencephalography studies. In effect, response activation (through the automatic direct way) and response correction also emerges from the analysis of the difference in electroencephalographic activity between the right and left hands (i.e. the lateralized readiness potential) during response selection. The lateralized readiness potential consists of an increase in electroencephalographic negativity over the motor cortex contralateral to the planned movement. In the non-corresponding condition during the Simon task, analysis of the lateralized readiness potential revealed an early deflection reflecting the automatic response initially activated by stimulus position, which is followed by a wave of opposite polarity corresponding to the activation of the correct response (Valle-Inclán, 1996; Vallesi et al., 2005; see also Leuthold, 2011).

The different effects obtained with PMd TMS in two different time windows is in line with previous results revealing that, during action selection and motor reprogramming, premotor regions exert an influence over the primary motor cortex that can rapidly change between facilitation and inhibition, depending on the behavioral context (Koch et al., 2006; O'Shea et al., 2007; Buch et al., 2010). O'Shea et al. (2007) investigated physiological interactions between the PMC and primary motor cortex with paired-pulse TMS during the execution of a visuo-motor choice RT task. The study reported that PMd TMS applied early after a cue to response selection facilitated primary motor cortex activity. Conversely, PMd TMS at a later time delayed choice RT. Similarly, Koch et al. (2006) showed that interhemispheric interactions between the left PMd and right primary motor cortex revealed both facilitatory and inhibitory connections at two subsequent times, as the PMd was involved not only in facilitating cued movements but also in suppressing movements that had been prepared. These results revealed that physiological interactions mediating response selection change on the basis of the behavioral context.

Our results are also in line with recent models of TMS effects. In TMS, the magnetic pulse causes the rapid and above-threshold depolarization of cell membranes affected by the current. This induced activity is not coherent with the ongoing activity of the neural population coding for a given stimulus or task, and depends on many factors (Miniussi et al., 2013). Recent studies explored the state-dependency effect of TMS by using a priming procedure (Cattaneo, 2010; Cattaneo et al., 2010). Here, stimulation had stronger effects on unprimed stimuli than on primed ones, indicating that, under this condition, TMS induces stronger activity in the less active neuronal population (Silvanto et al., 2008). Although the priming procedure was different from the task used here, a similar mechanism could be at the basis of our effects. TMS would have affected the correct response in a given trial (by slowing down corresponding or non-corresponding trials at different time points) by increasing activation in the neural population coding for the alternative response.

Finally, the finding presented here that the left, but not the right, PMd is involved in the Simon task is in line with previous data from neuroimaging and TMS studies showing that the PMd has a crucial role in tasks that emphasize response selection over response execution with a dominant role of the left hemisphere (Schluter et al., 2001; Johansen-Berg et al., 2002; Rushworth et al., 2003; O'Shea et al., 2007). Although it has been reported that the PMd is activated bilaterally during choice RT tasks, imaging and TMS studies suggested that the left PMd exerts dominance over the right (Schluter et al., 1998, 2001; Johansen-Berg et al., 2002). Functional imaging studies showed that, when subjects have to select between left and right hand movements, the right PMd is active only for movements executed by the left hand, whereas the left PMd is active for movements of either hand (Schluter et al., 2001). Similarly, TMS of the right PMd only disrupts the selection of left hand movements, whereas TMS of the left PMd disrupts the selection of movements that will be executed by either hand. By comparing interference at different times after presentation of the visual cue, it was possible to show that interference caused an effect earlier in the PMC than in the motor cortex (Schluter et al., 1998). This pattern of dominance is similar to that observed in apraxia, where left hemisphere lesions disrupt performance with the ipsilateral hand but right hemisphere lesions do not (Rushworth et al., 1998).

In conclusion, the present study contributes to our understanding of the temporal dynamics of the cortical network causally responsi- 
ble for elaborating responses in spatial stimulus-response conflict tasks. After the initial visuo-spatial preparation, the left PMd exerts its role in a two-fold manner: in a first activity, it selects the action with the highest stimulus-response compatibility, whereas the second activity appears only when, in non-corresponding trials, a conflict needs to be be resolved.

\section{Abbreviations}

AG, angular gyrus; PMC, premotor cortex; PMd, dorsal premotor cortex; RT, reaction time; SMG, supramarginal gyrus; SOA, stimulus onset asynchrony; TMS, transcranial magnetic stimulation.

\section{References}

Ashbridge, E., Walsh, V. \& Cowey, A. (1997) Temporal aspects of visual search studied by transcranial magnetic stimulation. Neuropsychologia, 35, $1121-1131$.

Buch, E.R., Mars, R.B., Boorman, E.D. \& Rushworth, M.F.S. (2010) A network centered on ventral premotor cortex exerts both facilitatory and inhibitory control over primary motor cortex during action reprogramming. J. Neurosci., 30, 1395-1401.

Cattaneo, L. (2010) Tuning of ventral premotor cortex neurons to distinct observed grasp types; a TMS-priming study. Exp. Brain Res., 207, 165172.

Cattaneo, Z., Devlin, J.T., Salvini, F., Vecchi, T. \& Silvanto, J. (2010) The causal role of category-specific neuronal representations in the left ventral premotor cortex (PMv) in semantic processing. Neurolmage, 49, 27282734.

De Jong, J.R., Liang, C.C. \& Lauber, E. (1994) Conditional and unconditional automaticity: a dual-process model of effects of spatial stimulusresponse correspondence. J. Exp. Psychol. Human., 20, 731-750.

Duqué, J., Labruna, L., Verset, S., Olivier, E. \& Ivry, R.B. (2012) Dissociating the role of prefrontal and premotor cortices in controlling inhibitory mechanisms during motor preparation. J. Neurosci., 32, 806-816.

Egner, T. \& Hirsch, J. (2005) The neural correlates and functional integration of cognitive control in a Stroop task. NeuroImage, 24, 539-547.

Egner, T., Delano, M. \& Hirsch, J. (2007) Separate conflict-specific cognitive control mechanisms in the human brain. NeuroImage, 35, 940-948.

Evans, A.C., Collins, D.L. \& Holmes, C.J. (1996) Toward a probabilistic atlas of human neuroanatomy. In Mazziotta, J.C. (Ed.), Brain Mapping: the Methods. Academic Press, San Diego, CA, pp. 343-361.

Forstmann, B.U., van den Wildenberg, W.P.M. \& Ridderinkhof, K.R. (2008) Neural mechanisms, temporal dynamics, and individual differences in interference control. J. Cognitive Neurosci., 20, 1854-1865.

Hommel, B. (1994) Spontaneous decay of response-code activation. Psychol. Res.-Psych. Fo., 56, 261-268.

Hommel, B. (2011) The Simon effect as tool and heuristic. Acta Psychol., 136, 189-202.

Iacoboni, M. \& Zaidel, E. (2004) Interhemispheric visuo-motor intergration in humans: the role of the superior parietal cortex. Neuropsychologia, 42, 419-425.

Johansen-Berg, H., Rushworth, M.F.S., Bogdanovic, M.D., Kischka, U., Wimalaratna, S. \& Matthews, P.M. (2002) The role of ipsilateral premotor cortex in hand movement after stroke. Proc. Natl. Acad. Sci. USA, 99, $14518-14523$.

Kerns, G. (2006) Anterior cingulate and prefrontal conrtex activity in an fMRI study of trial-by-trial adjustments on the Simon task. NeuroImage, 33, 399-405.

Koch, G., Franca, M., Del Olmo, M.F., Cheeran, B., Milton, R., Alvarez Sauco, M. \& Rothwell, J.C. (2006) Time course of functional connectivity between dorsal premotor and contralateral motor cortex during movement selection. J. Neurosci., 26, 7452-7459.

Kornblum, S., Hasbroucq, T. \& Osman, A. (1990) Dimensional overlap: cognitive basis for stimulus-response compatibility - a model and taxonomy. Psychol. Rev., 97, 253-270.

Leuthold, H. (2011) The Simon effect in cognitive electrophysiology: a short review. Acta Psychol., 136, 203-211.

Lu, C.H. \& Proctor, R.W. (1995) The influence of irrelevant location information on performance - a review of the Simon and spatial Stroop effects. Psychon. B. Rev., 2, 174-207.

Mapelli, D., Rusconi, E. \& Umiltà, C. (2003) The SNARC effect: an instance of the Simon effect? Cognition, 88, B1-B10.
Miniussi, C., Hrris, J.A. \& Ruzzoli, A. (2013) Modeling non-invasive brain stimulation in cognitive neuroscience. Neurosci. Biobehav. R., 37, 17021712.

Mochizuchi, H., Francsa, M., Huang, Y.-Z. \& Rothwell, J.C. (2005) The role of dorsal premotor area in reaction time task: comparing the 'virtual lesion' effect of paired pulse or theta burst transcranial magnetic stimulation. Exp. Brain Res., 167, 414-421.

O'Shea, J., Sebastian, C., Boorman, E.D., Johansen-Berg, H. \& Rushworth, M.F.S. (2007) Functional specificity of human premotor-motor cortical interactions during action selection. Eur. J. Neurosci., 26, 2085-2095.

Praamstra, P. (1999) Magnetic stimulation of the dorsal premotor cortex modulates the Simon effect. NeuroReport, 10, 3671-3674.

Ridderinkhof, K.R. (2002) Micro- and macro-adjustments of task set: activation and suppression in conflict tasks. Psychol. Res., 66, 312-323.

Ridderinkhof, K.R., van den Windelberg, W.P.M., Segalowitz, S.J. \& Cameron, S.C. (2004) Neurocognitive mechanisms of cognitive control: the role of prefrontal cortex in action selection, response inhibition, performance monitoring, and reward-based learning. Brain Cognition, 56, 129-140.

Rushworth, M.F., Nixon, P.D., Wade, D.T., Renowden, S. \& Passingham, R.E. (1998) The left hemisphere and the selection of learned actions. Neuropsychologia, 36, 11-24.

Rushworth, M.F., Ellison, A. \& Walsh, V. (2001) Complementary localization and lateralization of orienting and motor attention. Nat. Neurosci., 4, 656-661.

Rushworth, M.F., Johansen-Berg, H., Gobel, S.M. \& Devlin, J.T. (2003) The left parietal and premotor cortices: motor attention and selection. NeuroImage, 20(Suppl. 1), S89-S100.

Schiff, S., Bardi, L., Basso, D. \& Mapelli, D. (2011) Timing spatial conflict within the parietal cortex: a TMS study. J. Cognitive Neurosci., 23, 39984007.

Schluter, N.D., Rushworth, M.F., Passingham, R.E. \& Mills, K.R. (1998) Temporary interference in human lateral premotor cortex suggests dominance for the selection of movements. A study using transcranial magnetic stimulation. Brain, 121 (Pt 5), 785-799.

Schluter, N.D., Krams, M., Rushworth, M.F. \& Passingham, R.E. (2001) Cerebral dominance for action in the human brain: the selection of actions. Neuropsychologia, 39, 105-113.

Silvanto, J., Muggleton, N.G. \& Walsh, V. (2008) State-dependency in brain stimulation studies of perception and cognition. Trends Cogn. Sci., 12, $447-454$.

Simon, J.R. \& Rudell, A.P. (1967) Auditory S-R compatibility: the effect of an irrelevant cue on information processing. J. Appl. Psychol., 51, 300304.

Stürmer, B. \& Leuthold, H. (2003) Control over response priming in visuomotor processing: a lateralized event-related study. Exp. Brain Res., 153, $34-44$.

Stürmer, B., Leuthold, H., Soetens, E., Schroeter, H. \& Sommer, W. (2002) Control over location-based response activation in the Simon task: behavioral and electrophysiological evidence. J. Exp. Psychol. Human., 28, $1345-1363$.

Stürmer, B., Redlich, M., Irlbacher, K. \& Brandt, S. (2007) Executive control over response priming and conflict: a transcranial magnetic stimulation study. Exp. Brain Res., 183, 329-339.

Umiltà, C.A. \& Nicoletti, R. (1990) Spatial S-R compatibility. In Proctor, R.W. \& Rudell, A.P. (Eds), Stimulus-Response Compatibility: an Integrated Perspective. North Holland, Amsterdam, pp. 89-116.

Valle-Inclán, F. (1996) The locus of interference in the Simon effect: an ERP study. Biol. Psychol., 12, 147-162.

Vallesi, A., Mapelli, D., Schiff, S., Amodio, P. \& Umiltà, C. (2005) Horizontal and vertical Simon effect: different underlying mechanisms? Cognition, 96, B33-B43.

Wascher, E., Schatz, U., Kuder, T. \& Verleger, R. (2001) Validity and boundary conditions of automatic response activation in the Simon task. J. Exp. Psychol. Human., 27, 731-751.

Wassermann, E.M. (1998) Risk and safety of repetitive transcranial magnetic stimulation: report and suggested guidelines from the International Workshop on the Safety of Repetitive Transcranial Magnetic Stimulation, June 5-7, 1996. Electroen. Clin. Neuro., 108, 1-16.

van den Wildenberg, W.P., Wylie, S.A., Forstmann, B.U., Burle, B., Hasbroucq, T. \& Ridderinkhof, K.R. (2010) To head or to heed? Beyond the surface of selective action inhibition: a review. Front. Human Neurosci., 4, 222.

Wise, S.P., di Pellegrino, G. \& Boussaoud, D. (1996) The premotor cortex and nonstandard sensorimotor mapping. Can. J. Physiol. Pharm., 74, 469482. 